\title{
Les études aroumaines en Roumanie à l'heure européenne : quelques observations
}

The Aromanian Studies in Romania in the Age of the European Union: Some

Observations

Studiile aromâne în România la ceasul Europei: câteva observaŢii

Nicolas Trifon

\section{CpenEdition}

\section{Journals}

Édition électronique

URL : https://journals.openedition.org/ceb/9806

DOI : 10.4000/ceb.9806

ISSN : 2261-4184

Éditeur

INALCO

Édition imprimée

ISBN : 978-2-85831-239-9

ISSN : 0290-7402

Référence électronique

Nicolas Trifon, "Les études aroumaines en Roumanie à l'heure européenne : quelques observations », Cahiers balkaniques [En ligne], 44 | 2016, mis en ligne le 11 décembre 2017, consulté le 06 juillet 2021. URL : http://journals.openedition.org/ceb/9806 ; DOI : https://doi.org/10.4000/ceb.9806

Ce document a été généré automatiquement le 6 juillet 2021.

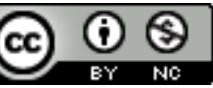

Cahiers balkaniques est mis à disposition selon les termes de la Licence Creative Commons Attribution - Pas d'Utilisation Commerciale 4.0 International. 


\title{
Les études aroumaines en Roumanie à l'heure européenne : quelques observations
}

\author{
The Aromanian Studies in Romania in the Age of the European Union: Some \\ Observations \\ Studiile aromâne în România la ceasul Europei: câteva observaŢii
}

Nicolas Trifon

1 Fin octobre 1997, lors des travaux du colloque intitulé les oubliés des Balkans qui se tenait à l'Inalco, rue de Lille, l'adresse aux organisateurs suivante a été lue par un participant :

Parmi les "oubliés » évoqués au cours de ce colloque, figurent les Aroumains, population dont la particularité est d'avoir conservé au cœur des Balkans une langue romane. Parlée sur le territoire de trois États (le sud de l'Albanie, la Macédoine ex-yougoslave et le nord de la Grèce) où elle n'a jamais bénéficié de véritable support scolaire ni de relais médiatique et éditorial, cette langue a connu une situation précaire tout au long de ce siècle [le $\mathrm{xx}^{\mathrm{e}}$ !]. Aujourd'hui, elle est menacée de disparition ${ }^{1}$.

2 La présence au colloque de deux intervenants a ensuite été critiquée. Il s'agissait d'Achille Lazarou ${ }^{2}$ et d'Antonis Koltsidas ${ }^{3}$, connus pour leur hostilité à l'enseignement de cette langue en Grèce, sous prétexte que "les Aroumains sont Grecs depuis toujours ", et qu'ils sont « racialement d'origine grecque » ou encore que leur idiome " relève d'un accident de l'histoire grecque ».

3 L'adresse, signée par l'Association des Français aroumains, s'intitulait "Sortir les Aroumains de l'oubli pour mieux les enterrer? » En la rédigeant, je ne pensais pas que quelques années plus tard des auteurs roumains, également d'origine aroumaine, et plus prestigieux que leurs confrères grecs, allaient prendre des positions autrement plus déconcertantes sur le plan intellectuel et se retrouver en matière de nationalisme, roumain cette fois-ci, sur des positions du même ordre. 


\section{Matilda Caragiu Marioțeanu : affirmations, ambiguïtés, « néoaroumanisme »?}

4 C'est lors d'une séance à l'Académie roumaine, le 28 janvier 2005, à Bucarest, qu'un événement mémorable s'est produit. Il marqua la rupture entre les promoteurs et animateurs du renouveau culturel aroumain dans la Roumanie de l'après-Ceauşescu, et « leurs » intellectuels, « leur » élite, c'est-à-dire des personnes réputées à la fois pour leurs travaux concernant les Aroumains et leur ascendance aroumaine. Retenu par d'autres obligations, je n'ai pas assisté à cette séance, pas plus qu'au colloque de l'Inalco en 1997.

5 La prestation de l'académicienne Matilda Caragiu Marioţeanu (1927-2009), référence majeure en matière de linguistique aroumaine, sur le thème « Les Aroumains : une crise identitaire ", a donné lieu à un réquisitoire de cette auteure contre tous ceux qui avaient mal interprété à ses yeux ses positions ou qui les avaient critiquées. Volontiers polémique, le discours prononcé en cette occasion, peut-être le moins académique de sa carrière, a été publié un an plus tard. C'est dans cette version écrite que sont formulées les notions de «néoaroumanisme» et de «néoaroumanistes» ou "néoaroumanisants $»^{4}$ pour désigner le mouvement qui aurait conduit à la crise identitaire des Aroumains et les responsables de ce mouvement, nommément cités. Entre-temps, en mai 2005, ces derniers avaient déposé une demande de reconnaissance des Aroumains comme minorité nationale en Roumanie.

La démonstration de Matilda Caragiu Marioţeanu comportait deux phases.

7 Dans un premier temps, elle rappelait et saluait les efforts déployés par ceux qui, au sein de la diaspora occidentale, autour notamment de Vasile Barba (1918-2007) de Fribourg-en-Brisgau, avaient publié des revues et des livres en aroumain, organisé des congrès et des stages de langue à partir de 1985, des publications et des manifestations auxquelles elle avait elle-même participé.

8 Dans un deuxième temps, elle accusait ces mêmes personnes d'avoir, petit à petit, " inculqué subtilement aux Aroumains de Roumanie, l'idée selon laquelle, d'un point de vue ethnique, ils seraient un autre peuple que les Roumains et que, du point de vue linguistique, leur langue serait différente du roumain parlé au nord du Danube ${ }^{5}$ ».

9 La violence de la charge menée contre ces personnes peut s'expliquer par le fait que Matilda Caragiu Marioţeanu a été, elle-même, accusée publiquement d'avoir soutenu que l'aroumain était une langue à part, après avoir dit que c'était un dialecte du roumain. Tout cela à cause de la première des douze "vérités » sur les Aroumains de son Dodécalogue, un texte culte paru en 1993: «Les Aroumains (Macédo-Valaques) et leur langue maternelle existent aujourd'hui et depuis deux mille ans ${ }^{6}$. »

10 Ces accusations, farfelues pour certaines, provenaient notamment de l'entourage de l'écrivain Hristo Cândroveanu (1928-2013), un auteur de facture traditionnelle apprécié surtout pour ses écrits en aroumain, directeur d'une publication financée par des fonds publics dans laquelle on vilipendait depuis 1990 tous ceux qui étaient suspectés de manquer de loyauté à l'égard de la nation roumaine; elles ne reposaient pas moins sur certains aspects quelque peu tortueux du raisonnement de Matilda Caragiu Marioţeanu. On peut les résumer comme suit : oui, l'aroumain est la langue maternelle des Aroumains, mais cela ne veut pas dire que l'aroumain soit une langue différente du roumain parce que le roumain est la langue littéraire, standard, 
des Aroumains. L'aroumain et le daco-roumain sont deux "hypostases» (variantes) d'une même langue, le proto-roumain, ou le roumain commun, ou ancien, issu du latin populaire, qui a connu une scission notamment après l'arrivée massive des Slaves dans le Sud-Est européen. Cette théorie, qui fait consensus parmi les linguistes roumains, est fondée sur une hypothèse cohérente, mais qui s'expose à plusieurs objections, la première étant que cela ne change rien au fait que les deux variantes ainsi que leurs locuteurs, les futurs Roumains et Aroumains, ont évolué séparément pendant un millénaire. Qu'il n'y ait pas une langue littéraire, standard, aroumaine, comme il y en a une pour le roumain, est facile à expliquer, puisque l'aroumain n'a jamais bénéficié d'un quelconque relais administratif ou scolaire; considérer que le roumain est la langue littéraire, standard, des Aroumains est un autre point. Elle l'est nécessairement en Roumanie, mais pas dans les autres pays où on parle l'aroumain et dont proviennent les Aroumains de Roumanie... Sur toutes ces questions, Matilda Caragiu Mariō̧eanu s'est bien gardée de se prononcer. Aussi, dans la conclusion de son discours à l'Académie, se contente-t-elle de reprendre, sur un ton encore plus sentencieux, sa vérité du Dodécalogue :

Le principal but de chaque Aroumain, qu'il soit guidé par des principes traditionnels ou par de nouveaux principes, n'est autre que la conservation de l'aroumain, notre langue maternelle : que cette langue ne se perde pas, qu'elle soit conservée comme une précieuse réserve pour l'(a)roumanité, la romanité, la balkanité. Car les Aroumains ne peuvent être sauvés que par leur langue maternelle. Sans ce certificat de naissance, on ne pourra plus parler d'Aroumains ${ }^{7}$.

11 On peut penser ce que l'on veut de l'ambiguïté cultivée à maintes reprises par Matilda Caragiu Marioţeanu sur ce point, y compris la suspecter ou l'accuser de ne pas assumer jusqu'au bout le credo national roumain qu'elle affiche par ailleurs. Pour ma part, je vois dans cette ambiguïté plutôt une ouverture à propos de la question, forcément complexe, des rapports entre le roumain et l'aroumain. Prétendre la résoudre en tranchant purement et simplement est illusoire.

12 En revanche, une chose est sûre, c'est qu'avec cette intervention, Matilda Caragiu Marioţeanu a fourni un argument en or à ceux qui n'avaient que faire de telles subtilités et qui ne cherchaient qu'à trancher une fois pour toutes en se contentant de répéter à l'envi: l'aroumain est un dialecte, les Aroumains sont Roumains, l'Académie l'a dit, c'est scientifique. Elle a ainsi donné le feu vert au retour à la version "traditionnelle " à propos des Aroumains, forgée au lendemain de la création de l'État moderne roumain (1859) à l'occasion de l'assistance culturelle et de la protection politique qu'il a assurées à partir de 1864 aux Aroumains de l'Empire ottoman qui s'opposaient aux assauts du nationalisme grec à la veille du partage de la Turquie d'Europe entre les États-nations balkaniques. Cette version, bien enracinée dans le corpus national roumain, venait d'être malmenée par une nouvelle lecture critique de l'histoire et surtout par l'irruption des revendications identitaires aroumaines après 1990, au grand dam de ceux qui, dans la foulée de la critique du communisme, s'accrochaient aux valeurs d'antan et tendaient à réhabiliter la politique menée sous les régimes précédents.

\section{Matilda Caragiu Marioțeanu : pourquoi cette position?}

13 Les temps avaient changé, pas les velléités de grandeur nationale, même si les objectifs étaient, en l'occurrence, dérisoires. Depuis quelques années, le département des 
«Roumains de partout» auprès du gouvernement roumain, ses experts, ses relais universitaires et médiatiques, préparent un nouveau front pour faire reconnaître, grâce aux Aroumains, l'existence d'une minorité roumaine en Albanie et en République de Macédoine, la Grèce étant un trop gros morceau pour tenter quelque chose. Autant dire que les nouvelles revendications spécifiques aroumaines faisaient désordre, et mettaient dans l'embarras surtout les personnalités publiques dont la réputation était d'une manière ou d'une autre associée à leur ascendance aroumaine, ascendance qui a souvent constitué plutôt un atout en Roumanie. Parmi ces personnalités, les linguistes et les historiens allaient être appelés à jouer un rôle de premier plan.

$14 \mathrm{Au}$ lendemain même de la prestation de Matilda Caragiu Marioţeanu, la linguiste Mariana Bara (1961-), prenait position dans un texte ayant pour titre «Crise de l'identité ou crise de la description des Aroumains? » La crise d'identité des Aroumains est due à l'inadéquation entre le discours officiel et les réalités aroumaines telles qu'on peut les observer de nos jours en Roumanie et dans les Balkans, faisait-elle remarquer. Et elle dénonçait l'attitude de ceux qui refusent de procéder à la réévaluation critique d'un discours forgé au XIX siècle, avec les critères du $\mathrm{XIX}^{\mathrm{e}}$ siècle et presque exclusivement en Roumanie ${ }^{8}$.

Quelque dix ans après avoir été exposée, cette prise de position critique demeure isolée en Roumanie. En revanche, la reprise pure et simple de l'assertion «l'aroumain est un dialecte du roumain " est devenue monnaie courante. Plus étonnant encore est le silence observé par nombre de personnes qui étaient à même d'intervenir dans ce genre de débat.

Les raisons qui ont poussé Matilda Caragiu Marioţeanu à trancher sur un ton aussi catégorique tout en cultivant le paradoxe et en entretenant ainsi le suspense sont nombreuses et aucune ne se suffit à elle-même. Ces raisons valent aussi pour ceux qui ont repris à leur compte les accusations proférées par la linguiste ou qui se sont abstenus de les critiquer, jugeant la question trop sensible. En voici cinq :

1. À la veille de sa performance relatée plus haut, Matilda Caragiu Marioțeanu venait d'être promue membre titulaire de l'Académie roumaine (en 2004) dont elle n'était que membre correspondante auparavant. Il s'agissait de la reconnaissance suprême que l'on puisse obtenir en Roumanie sur le plan institutionnel. Sans doute remplissait-elle les critères, mais comment courir le risque de se voir reprocher son ingratitude ou encore de faire l'objet de campagnes calomnieuses? Cette explication vaut aussi pour d'autres personnes qui n'étaient guère disposées non plus à sacrifier leur carrière ou leur réputation en prenant des positions à contre-courant ;

2. Matilda Caragiu Marioțeanu, et pas seulement elle, aurait-elle subi des pressions, directes ou indirectes, en raison, justement, de son parcours sans faute à l'époque communiste? Ce genre d'explication, avancée souvent, mais sans preuve, dans les anciens pays communistes, est peu probable ;

3. Il faut en revanche prendre en compte la difficulté de Matilda Caragiu Marioțeanu de communiquer ou plutôt de s'entendre avec ces nouveaux venus qui avaient le vent en poupe en raison des succès enregistrés par la dynamique associative culturelle des années 1990 et avec les personnes de la diaspora qui avaient en quelque sorte préparé et accompagné cette dynamique. Cette difficulté semble avoir joué un rôle central dans la réaction de la linguiste. Il en va de même pour le directeur de l'Institut de dialectologie rattaché à l'Académie, 
Nicolae Saramandu (1941-), qui s'était pourtant beaucoup engagé dans le renouveau culturel des années 1990, mais qui a fini par prendre ses distances ${ }^{9}$. En effet, les ténors de la diaspora n'étaient pas des spécialistes en sciences humaines, mais des ingénieurs, ou des membres des professions libérales, tandis que la structure socioprofessionnelle des animateurs du mouvement et de leur public en Roumanie était très hétéroclite, même si les "littéraires", souvent de fraîche date, c'est-à-dire les personnes qui avaient commencé à écrire en aroumain à partir de cette date, étaient nombreux. En simplifiant à l'extrême, on pourrait dire que ces derniers disaient aux premiers: "Vous êtes des spécialistes reconnus et nous sommes fiers de vous, appuyez nos demandes, vous êtes Aroumains comme nous, vous devez donc le faire ! » À ceci, les « intellectuels » rétorquaient : « Vous êtes des amateurs en matière de linguistique et d'histoire, laissez-nous faire, nous les spécialistes ${ }^{10}$ !» Cet argument d'autorité passait mal et c'est ainsi que de sous-entendu on est passé au malentendu, de la cacophonie à la rupture. À peine un an après l'entrée en scène des accusations de "néoaroumanisme », comme pour les confirmer, plusieurs chefs de file des "accusés " se lançaient dans une tentative pour le moins hasardeuse d'enraciner les revendications aroumaines dans un territoire moyennant des références imaginaires aux Macédoniens de l'Antiquité. Sous le nom de makedonarmân («macédonarman », en français), un nationalisme aroumain venait ainsi de faire son apparition; dans l'optique de ses promoteurs, les Macédoniens d'Alexandre le Grand sont appelés à jouer le même rôle fondateur que celui attribué aux Daces dans le discours national roumain ${ }^{11}$;

4. Les convictions nationalistes, telles qu'elles sont affichées à tout va en Roumanie, y compris dans les milieux intellectuels, ne semblent pas avoir été déterminantes dans les prises de position de personnes aussi pondérées et rationnelles que Matilda Caragiu Marioţeanu, Nicolae Saramandu, ou encore d'un historien comme Nicolae şerban Tanaşoca (1941-), spécialiste des périodes byzantine et ottomane et auteur de nombreux travaux sur les Aroumains. En revanche, il est indéniable que leurs prises de position peuvent être qualifiées en dernière instance de nationalistes.

Les auteurs qui se réclament à cor et à cri des valeurs nationales ne manquent évidemment pas. Sur le plan des motivations, le nationalisme joue un rôle significatif dans leur engagement, même si d'autres facteurs, tels que les considérations liées à la carrière ou à la réputation, doivent être pris en compte. Il en va ainsi pour l'écrivain Hristo Cândroveanu, cité plus haut ${ }^{12}$, ou encore pour l'historien Stoica Lascu (1951-), de l'université Ovidiu de Constanţa, auteur de travaux très pointilleux sur divers aspects de l'histoire des Aroumains, mais dont la rhétorique accusatrice sort tout droit du corpus national-communiste de l'époque Ceauşescu $^{13}$. Les convictions nationalistes jouent un rôle considérable chez des historiens et politologues non aroumains : le chercheur Emil ţîrcomnicu (1971-), est celui qui est allé le plus loin dans la politisation de la question aroumaine à partir $\mathrm{du}$ discours de Matilda Caragiu Marioțeanu ${ }^{14}$. Cela peut s'accompagner de véritables déclarations d'amour dont on ne saurait a priori nier la sincérité. Dernier exemple en date, le dossier publié dans le magazine Historia daté de mai 2014 par Marius Diaconescu (1970-), sous le titre « Qui sont les Aroumains ${ }^{15}$ ? » L'académicien Dan Berindei (1923-), historien réputé, qui a accompagné différentes manifestations pro-roumaines à Bucarest et à Tirana ces dernières années, est plus réservé. En règle générale, les auteurs roumains s'appuient sur leurs confrères d'origine aroumaine pour se positionner sur les questions sensibles ${ }^{16}$. Le cas de Gheorghe Zbuchea (1940-2008) est plus compliqué. Avec Stelian Brezeanu (1941-), il a exhumé et édité toute une série de documents qui permettent une compréhension plus nuancée de la politique menée par l'État roumain par rapport aux Aroumains au cours de l'histoire moderne, ce qui ne l'a pas empêché de les présenter et de les commenter dans une perspective nationaliste ${ }^{17}$.

Évidemment, il n'est pas facile - et le risque de se perdre en conjectures oiseuses est 
grand - de faire la part de ce qui relève de la conviction nationaliste et des soucis concernant la carrière et la notoriété des auteurs, surtout lorsqu'il s'agit des plus jeunes d'entre eux. La question des débouchés des travaux sur les Aroumains et de leur langue, qui n'est d'ailleurs enseignée que dans un cadre très restreint, ne saurait être éludée. Comme objet de recherche, ils présentent un certain intérêt, et la multiplication des études linguistiques le montre. Scientifiquement, ces études, qui s'appuient sur de longues recherches de terrain entreprises dans le cadre de l'Académie roumaine, sont irréprochables. Leurs auteurs se gardent bien, en revanche, de remettre en question la version de l'Académie, présentée comme officielle, sur les rapports entre l'aroumain et le roumain. Les autres débouchés à proprement parler sont très rares. C'est à la réputation de "super-roumains » que l'on doit les avantages qui peuvent se présenter à ceux qui s'y intéressent. La position la plus «valorisante " que l'on puisse occuper est celle de «spécialiste » des Aroumains, conseiller au ministère des Affaires étrangères, ou encore dans la diplomatie. C'est le cas de l'ambassadeur de la Roumanie à Tirana, Viorel Stănila (1968-), membre, par ailleurs, de l'Institut d'études sud-est européennes auprès de l'Académie, qui est intervenu publiquement en mars 2013 pour demander la fermeture d'une exposition sur le livre aroumain à la Bibliothèque nationale à cause de l'attitude jugée antiroumaine des organisateurs ${ }^{18}$. C'est également dans l'orbite du département des «Roumains de partout » que gravite une fondation du même nom qui subventionne la publication d'ouvrages et l'organisation de manifestations sur les Aroumains avec la participation d'universitaires contents de trouver des moyens matériels leur permettant d'avancer dans leurs travaux et de se faire connaître $^{19}$;

5. Dans bien des cas, une sorte de conservatisme, le respect dû aux anciens et à leur choix, courant chez les Aroumains, et dans la zone dont ils proviennent, semble jouer un rôle décisif. Les parents ou grands-parents ayant décidé de venir en Roumanie, ou s'étant engagés dans les Balkans au service de l'État roumain, il n'est pas facile de douter rétrospectivement de leur choix. C'est à ce devoir de respect, que l'on doit les exagérations de l'acteur Ion Caramitru (1941-), ancien ministre de la Culture, à la tête de la Société de culture macédo-roumaine, principal pourfendeur ces dernières années des «néoaroumaniste ${ }^{20}$ ».

On arrive ainsi à une explication plus « historique » que sociale et psychologique de la rupture survenue entre des gens qui ont fait tant de choses ensemble avant de s'entre-déchirer. Les initiatives de la diaspora aroumaine visaient la question aroumaine dans les Balkans en général. Nicolae Saramandu, surtout, et aussi Matilda Caragiu Marioţeanu, se sont impliqués dans les activités de la diaspora qui relevaient, selon cette dernière, d'une « action pour la sauvegarde des Aroumains dont l'existence était menacée ${ }^{21}$ ». Le principal pays concerné était la Grèce. Tous les pays balkaniques étaient représentés lors des congrès et dans les publications, mais les organisateurs et les principaux auteurs des initiatives étaient originaires de Roumanie. Fribourg-en-Brisgau était d'ailleurs un des centres culturels de l'exil culturel roumain et dans les publications d'excellente qualité de la bibliothèque roumaine de cette ville de nombreuses études sur les Aroumains avaient paru avant 1985 ; à cette date, les activités des uns et des autres se sont séparées, l'Union pour la culture et la langue aroumaine fondée par Vasile Barba ayant mis en place sa propre bibliothèque à l'Université grâce au soutien de la section des langues romanes de cet établissement. L'implosion du communisme en Roumanie et en Albanie, ainsi que l'éclatement de la Fédération yougoslave, allaient donner un écho particulier à ces initiatives un peu partout, mais surtout en Roumanie, le pays dans lequel il y avait de loin le climat le plus favorable pour eux et leurs doléances. Ce fut cependant autre chose qui allait créer la surprise. Il s'agit de l'évolution de l'attitude collective des Aroumains de Roumanie, qui 
avaient mieux conservé leur langue ${ }^{22}$ que ceux de Bulgarie, de Grèce et d'Albanie, pays qu'ils avaient quittés pendant la période 1925-1932. Ils ont formulé avec une détermination toute particulière les revendications concernant les droits des minorités, des revendications conçues par la diaspora surtout pour les Aroumains vivant dans les pays autres que la Roumanie. Pris de court par le décalage entre la dynamique roumaine et la situation peu encourageante qui prévalait dans les autres pays, à commencer par la Grèce, certains, y compris Vasile Barba, s'inquiétèrent à l'idée que si on revendique un tel statut en Roumanie, on perde le soutien de ce pays, le seul qui ait manifesté un réel intérêt pour les Aroumains. Bien qu'ils aient pris leurs distances avec la Roumanie, notamment en mettant systématiquement en avant la langue aroumaine et en abandonnant toute référence à ce pays dans les actions promues dans les Balkans, ils n'étaient pas prêts à l'aventure que représentait la demande du statut de minorité nationale en Roumanie. Mais c'était trop tard. Dans un sens, ce rapport à la loyauté, cette crainte de manquer au devoir de réserve que l'on observe chez ceux qui avaient au départ travaillé pour la Roumanie, ou qui ont été formés, et ont prospéré dans ce pays, n'est pas sans faire penser à l'époque ottomane. Enfin, l'esprit européen n'était pas au rendez-vous: on hésite encore en Roumanie comme dans les autres pays des Balkans à revendiquer un droit, aussi justifié soit-il ${ }^{23}$, quand on ne peut pas compter sur une autorité à même de le faire valoir. De ce point de vue, nombre de descendants des colons de l'entre-deux-guerres, plus terre-à-terre et véhiculant parfois des idées saugrenues, ont mieux compris que les temps avaient changé.

Autant dire que les études aroumaines sont devenues tout aussi problématiques en Roumanie qu'elles l'étaient (et qu'elles le sont encore, mais de manière plus apaisée) dans un pays comme la Grèce. Le nationalisme sous ses différentes formes et la politisation excessive de la question aroumaine continuent de brouiller les pistes en empêchant l'établissement d'un consensus minimal autour de la langue et de la culture de cette population.

Quels sont les changements survenus depuis 1990 dans le domaine aroumain? L'examen de la bibliographie pour la période 1990-2004 rédigée par le géographe Thede Kahl (1971-), sans doute le meilleur spécialiste en la matière ${ }^{24}$, permet de pointer plusieurs aspects significatifs :

1. Certes, c'est en Roumanie que l'on a publié le plus, mais le décalage par rapport aux autres pays, notamment la Grèce et la Macédoine, s'est réduit ;

2. L'aspect le plus remarquable est l'accroissement vertigineux du nombre de livres et périodiques en aroumain. Là encore, même en comparaison avec la Macédoine où l'aroumain est reconnu officiellement, la Roumanie se situe en tête. Pendant cette période, la maison d'édition privée Cartea aromână a publié, aux États-Unis (Fayetteville, NY) tout d'abord, puis à Constanţa, 69 titres, la plupart en aroumain, tandis que la FundaŢia culturală aromână de Bucarest, subventionnée par l'État, a publié 31 titres dont certains en aroumain. À Skopje, la Bibliothèque nationale aroumaine a publié 40 livres. En Grèce, le nombre de ce type de publications est très réduit ;

3. 11 y a un autre aspect qui mérite d'être relevé : la production «scientifique " a marqué le pas par rapport à la période communiste. Lorsque l'on regarde de plus près les principaux auteurs qui figurent dans les rubriques «Langue " et "Histoire », force est de constater que leurs premiers et parfois plus importants travaux publiés dans des revues de spécialité remontent aux années précédant la chute du communisme. Il suffit de prendre comme repère la Bibliographie 
macédo-roumaine parue à Fribourg fin 1984 sous la direction du byzantiniste Petre Năsturel pour le constater. Matilda Caragiu Marioţeanu est 20 fois référencée (depuis 1958), Nicolae Saramandu, 16 fois (depuis 1974), Elena Scărlătoiu, 7 fois (depuis 1968), Nicolae-şerban Tanaşoca, 4 fois (depuis 1973), etc.

\section{Conclusion}

20

Enfin, en guise de conclusion, voici plusieurs observations sur les champs couverts par les travaux référencés et les tendances actuelles.

Les travaux des linguistes portent surtout, voire exclusivement, sur la langue aroumaine, alors que ceux des historiens qui figurent dans la bibliographie aroumaine ne concernent parfois qu'indirectement les Aroumains. C'est dire la place stratégique qu'occupent les linguistes. Traditionnellement, ce sont eux qui ont établi la date de la séparation du roumain de l'aroumain ${ }^{25}$. En revanche, les historiens se sont en général montrés plus prudents sur ce point, les conclusions auxquelles conduisaient les critères linguistiques n'étant pas confirmées par des preuves historiques.

De nos jours, les historiens conséquents refusent de s'aventurer dans des affirmations catégoriques sur ces problèmes et évitent le mélange des genres ${ }^{26}$. Malheureusement, nombre d'autres auteurs, y compris parmi ceux appartenant aux jeunes générations, préfèrent, par conviction ou par opportunisme, s'en tenir scrupuleusement à la version " officielle » roumaine qui résulte pour l'essentiel des raisonnements linguistiques ${ }^{27}$.

23 Cependant, le nationalisme fait de moins en moins la loi dans l'Université en Roumanie, les chercheurs voyagent de plus en plus et suivent de près les changements survenus en Occident dans leurs disciplines. Au sein de la jeune génération d'historiens, des voix nouvelles se font entendre. En conclusion de sa thèse de doctorat soutenue en 2007 et publiée en 2009 sous le titre le " problème aroumain » dans les rapports de la Roumanie avec les États balkaniques (1900-1913), l'historien IonuȚ Nistor (1980-) de l'université de Iaşi n'hésite pas à écrire :

L'intérêt pour le sort de cette ethnie était dû, à notre avis, non pas tant à l'intention de protéger les droits des Aroumains, et de leur garantir une meilleure situation parmi les sujets chrétiens du sultan de Constantinople, qu'au rôle que les Aroumains pouvaient jouer dans les Balkans et aux conséquences de la «solution » $\mathrm{du}$ problème aroumain pour la diplomatie roumaine. [...] Le destin de la communauté aroumaine n'intéressait le gouvernement de Bucarest que dans la mesure où il servait ses objectifs politico-territoriaux ${ }^{28}$.

On peut également citer, pour ce qui est de l'après-1913, les historiens d'origine aroumaine Enache Tuşa (1969-), avec son livre sur la Dobroudja et la colonisation des Aroumains dans le sud de cette province ${ }^{29}$, et Alexandru Gica (1969-), avec son histoire récente des Aroumains en Roumanie présentée dans l'annuaire du New Europe College de Bucarest ${ }^{30}$.

Les anthropologues et ethnologues qui s'intéressent aux Aroumains depuis 1990 occupent une place à part. Il s'agit de disciplines qui ont pris le relais des études sur le folklore notamment, en lien direct avec les écoles nord-américaines et occidentales. Ces auteurs sont moins enclins à juger, se contentant souvent de relativiser. Leur poids critique par rapport à l'imaginaire roumain demeure réduit, même si la situation a changé. Pour ce qui est des Aroumains, le point de départ a été donné par Irina Nicolau (1946-2002), auteure en 1993 d'une thèse retentissante sur les "caméléons des Balkans $^{31} »$. Elle a ouvert une voie dans laquelle de jeunes auteurs, telle Corina Iosif 
(1960-), qui a soutenu sa thèse de doctorat intitulée l'Entreprise de la parenté: réseaux d'échanges entre les Aroumains de ConstanŢa à l'École des hautes études en sciences sociales en $2007^{32}$, semblent s'inscrire avec bonheur.

\section{BIBLIOGRAPHIE}

BARA Mariana, 2005, „Criză de identitate sau criza descrierii”, Daima, I, 1, pp. 10-12.

BREZEANU Stelian et ZBUCHEA Gheorge (ed.), 1997, Românii de la Sud de Dunăre. Documente, Bucureşti: Editura Arhievele NaŢionale ale României. URL : http://www.proiectavdhela.ro/pdf/ gheorghe_zbuchea_romanii_la_sud_de_dunare.pdf (consulté le 13 mars 2014).

BREZEANU Stelian, 1999, O istorie a românilor din Peninsula Balcanică: secolele XVIII-XX, Bucureşti: Editura Biblioteca Bucureştilor.

URL : https://arhiva.bibmet.ro/Uploads/3_2011/154006.pdf (consulté le 13 mars 2014).

CARAGIU MARIOŢEANU Matilda, 2006, Aromânii şi aromâna în conştiinţa contemporană [Les Aroumains et l'aroumain dans la conscience contemporaine], București: Editura Academiei Române.

CARAGIU MARIOŢEANU Matilda, 1993, „Un dodecalog al Aromânilor sau 12 adevăruri incontestabile, istorice şi actuale, asupra aromânilor şi asupra limbii lor" [Un Dodécalogue ou 12 vérités incontestables, historiques et actuelles sur les Aroumains et leur langue], România literară, vol. XXVI, nr. 33 ; trad. fr. parue en 1998 in MicRomania : Littératures en langues romanes de moindre expansion, $\mathrm{n}^{\circ} 26-3$.

GICA Alexandru, 2009, “The Recent History of the Aromanians in Romania”, in New Europe College Yearbook 2008-2009, Bucharest: New European College, pp. 173-200. URL : http://www.nec.ro/data/ pdfs/publications/nec/2008-2009/ALEXANDRU_GICA.pdf (consulté le 13 mars 2014).

IOSIF Corina, 2013, « Frontières réelles, frontières imaginaires : l'identité aroumaine au début du $\mathrm{XX}^{\mathrm{e}}$ siècle », in Gilles ROUET et François sOULAGES (dir.), Frontières géoculturelles \& géopolitiques, Paris : L'Harmattan, p. 133-147.

KAHL Thede, 2006, Istoria aromânilor, Bucureşti: Trictonic.

KOLTSIDAS Antonis, 1998, «L'état présent de la langue koutsovalaque sur le territoire de la Grèce », Cahiers balkaniques, $\mathrm{n}^{\circ} 25$, p. 99-127.

LAZAROU Achille, 1986, l'aroumain et ses rapports avec le grec, Thessalonique : Institut des Études balkaniques.

NICOLAU Irina, 1993, « Les caméléons des Balkans », Civilisations, vol. XIII, nº 2, p. 175-178.

NISTOR IonuȚ, 2009, „Problema aromână” în raporturile României cu statele balcanice (1903-1913) [Le « problème aroumain » dans les rapports de la Roumanie avec les États balkaniques (1900-1913)], Iaşi: Editura Universităţii Alexandru Ioan Cuza.

SAMARANDU Nicolae, 1988, „Harta graiurilor aromâne şi meglenoromâne din Peninsula balcanică” [Carte des parlers aroumains et méglénoroumains de la péninsule balkanique], Studii şi cercetări lingvistice, anul XXXIX, nr. 3, pp. 225-245. 
TîTRCOMNICU Emil, 2011, „Aromânii din România, astăzi”, Revista română de sociologie, seri nouă, anul XxII, nr. 1-2, pp. 153-169. URL : http://www.revistadesociologie.ro/pdf-uri/nr.1-2-2011/08ETircomnicu.pdf (consulté le 13 mars 2014).

TRIFON Nicolas, 2007, « Les Aroumains en Roumanie depuis 1990 : comment se passer d'une (belle-)mère patrie devenue encombrante ", Revue d'études comparatives Est-Ouest, vol. 38, $\mathrm{n}^{\circ} 4$, p. 173-199. URL : http://www.persee.fr/doc/receo_0338-0599_2007_num_38_4_1868 (consulté le 13 mars 2014).

TUşA Enache, 2011, Imaginar politic şi identităŢi colective în Dobrogea, București: Institutul de ştiinţe politice şi relaŢii internaŢionale.

\section{NOTES}

1. Denise EeCKAUte-BARDery (dir.), 2000, les oubliés des Balkans: comptes rendus, suppléments, Cahiers balkaniques, $\mathrm{n}^{\circ} 30$, p. 74.

2. Auteur notamment en 1986 de l'aroumain et ses rapports avec le grec, Thessalonique : Institut des Études balkaniques et, en 1996 de les Valaques et l'Union européenne, Thessalonique : Institut des Études balkaniques.

3. «La langue koutsovalaque est sur une pente descendante. [...] Cela n'a pas de sens de reconstituer cette langue, ni même de l'enseigner ", concluait dans sa contribution cet auteur. Voir Antonis KOLTSIDAS, 1998, «L'état présent de la langue koutsovalaque sur le territoire de la Grèce ", Cahiers balkaniques, $\mathrm{n}^{\circ}$ 25, p. 122.

4. Matilda CARAGIU MARIOŢEANU, 2006, Aromânii şi aromâna în conştiinŢa contemporană [Les Aroumains et l'aroumain dans la conscience contemporaine], Bucureşti: Editura Academiei Române, pp. 58-80.

5. Ibid., p. 63.

6. Matilda CARAGIU MARIOŢEANU, 1993, „Un dodecalog al Aromânilor sau 12 adevăruri incontestabile, istorice şi actuale, asupra aromânilor şi asupra limbii lor" [Un Dodécalogue ou 12 vérités incontestables, historiques et actuelles sur les Aroumains et leur langue], in România literară, vol. xxvI, nr. 33 ; la traduction française est parue en 1998 dans MicRomania: Littératures en langues romanes de moindre expansion, $\mathrm{n}^{\circ}$ 26-3, Charleroi (Belgique).

7. Ibid., p. 79.

8. Mariana BARA, 2005, „Criză de identitate sau criza descrierii”, Daima, I, 1, pp. 10-12. Pour le compte rendu de la séance de l'Académie, voir aussi Nicolas TRIFON, 2007, « Les Aroumains en Roumanie depuis 1990 : comment se passer d'une (belle-)mère patrie devenue encombrante ", Revue d'études comparatives Est-Ouest, vol. 38, n 4, p. 173-199. URL : http://www.persee.fr/doc/receo_0338-0599_2007_num_38_4_1868 (consulté le 13 mars 2014).

9. Auteur de nombreux ouvrages et contributions, le linguiste Nicolae Saramandu s'était par ailleurs impliqué dans diverses activités, notamment à Fribourg aux côtés de Vasile Barba. Tout en rédigeant l'abécédaire aroumain destiné aux cours facultatifs d'aroumain en République de Macédoine paru à Skopje en 1997, il a assuré la formation des journalistes de l'émission en aroumain de Radio România Internaţional, qui émet depuis mars 1991, et a participé aux travaux de standardisation de l'aroumain à Bitola 
en 1997. Parmi ses travaux, citons : Nicolae SAMARANDU, 1988, „Harta graiurilor aromâne şi meglenoromâne din Peninsula Balcanică" [Carte des parlers aroumains et méglénoroumains de la péninsule balkanique], Studii şi cercetări lingvistice, anul Xxxıx, nr. 3, pp. 225-245. Sur la question langue/dialecte, il ne s'est pas prononcé dans la presse spécialisée, se contentant de prendre position publiquement lors de l'émission „Naşul” [Le Parrain] modérée par Radu Moraru, sur ZeceTV le 11 juillet 2011, aux côtés de l'acteur Ion Caramitru, de l'historien Nicolae-şerban Tanaşoca et de l'académicien Dan Berindei.

10. Dans un sens, c'est une "trahison des clercs » (dans le genre de celle décriée par Julien Benda dans son livre paru en 1929 sous ce titre) qui semble se jouer du côté des «spécialistes ». Tout en se réclamant de la science et se présentant comme détenteurs désintéressés de la vérité, Matilda Caragiu MarioŢeanu et ses confrères n'hésitent pas à descendre dans l'arène, à s'engager, à prendre position.

11. Kira Mantsu IORGOVEANU, Mihali PREFTI et Gica GODI, 2007, in Bana armânească, $\mathrm{n}^{\circ} 1-2$ (47/48), pp. 16-18.

12. Dans un mémoire adressé le 20 janvier 2003 au gouvernement roumain intitulé « La revitalisation de la Société de culture macédoroumaine est dans l'intérêt de l'État roumain ", il attire l'attention sur l'inefficacité des actions menées à cette date en faveur de la diffusion de l'idée roumaine parmi les Aroumains des Balkans et, par la même occasion, dénonce les "activités antiroumaines" de certaines associations aroumaines de Roumanie. Dans un texte rédigé en aroumain il va jusqu'à souhaiter que dans "nos écoles", celles que la Roumanie devrait ouvrir dans les Balkans, l'enseignement n'ait pas lieu en aroumain mais en roumain. Voir Hristu CÂNDROVEANU, 1995, Aromânii ieri şi azi: cutură, literatură, probleme, perspective, Craiova: Scrisul Românesc, pp. 40-42.

13. Avec Nistor Bardu, il se prononce par exemple contre les « actions antinationales et anhistoriques [...] des représentants de la soi-disant communauté aroumaine », ces " roublards " motivés par « des intérêts explicitement pécuniaires ", et s'inquiète de l'intérêt que pourrait leur accorder le «trop tolérant » État roumain. Voir Stoica LASCU et Nistor BARDU (ed.), 2005, „Aromânii nu sunt minoritari în România” [Les Aroumains ne sont pas des minoritaires en Roumanie], Cuget liber, 28 décembre.

14. Emil ŢîRcomNicU, 2011, „Aromânii din România, astăzi”, Revista română de sociologie, seri nouă, anul XXII, nr. 1-2, pp. 153-169. URL : http://www.revistadesociologie.ro/pdfuri/nr.1-2-2011/08-ETircomnicu.pdf (consulté le 13 mars 2014).

15. Marius DIACONESCU, 2004, „Cine sunt arômanii?” [Qui sont les Aroumains ?], Historia, nr. 148, pp. 12-33.

16. En recevant Matilda Caragiu Marioţeanu le 28 janvier 2005, le président de l'Académie, Eugen Simion, faisait remarquer dans sa présentation que, pour « l'imaginaire collectif [roumain], l'Aroumain, ce frère méridional, est rentré chez lui tel le fils prodigue, un retour plutôt contraint, entre nous soit dit. Il déblatère contre tous ou, comment le dire, il a une langue de vipère. Mieux vaut donc ne pas le contrarier et, si on le fait, il faut s'attendre à une brouille pour la vie. »

17. Stelian BREZEANU et Gheorghe ZBUCHEA (ed.), 1997, Românii de la Sud de Dunăre. Documente, București: Editura Arhievele NaŢionale ale României. URL: http:// www.proiectavdhela.ro/pdf/gheorghe_zbuchea_romanii_la_sud_de_dunare.pdf (consulté le 13 mars 2014); Gheorghe ZBUCHEA, 1999, 0 istorie a românilor din Peninsula 
Balcanică: secolele XVIII-XX, Bucureşti: Editura Biblioteca Bucureştilor. URL : https:// arhiva.bibmet.ro/Uploads/3_2011/154006.pdf (consulté le 13 mars 2014).

18. URL : http://www.cotidianul.ro/va-reusi-ambasadorul-romaniei-in-albania-sainchida-astazi-o-expozitie-cu-initiativa-privata-208678/

19. Voir par exemple Adina BERCIU-DRĂGHICESCU, 2010, Aromânii din Albania: prezervarea patrimoniului lor imaterial: proiect realizat sub egida Bibliotecii Metropolitane București şi a Departamentului Pentru Românii de Pretutindeni de pe lângă Guvernul României, Bucureşti: Editura Biblioteca Bucureştilor. Historienne, née en 1950, la coordinatrice de ce projet est l'auteure de plusieurs ouvrages sur les Aroumains/Roumains du Sud parus ces dernières années avec le soutien de la fondation susnommée.

20. Ion Caramitru était au centre du talk-show télévisé du 11 juillet 2011. Voir supra, note 11.

21. Matilda CARAGIU MARIOŢEANU, 2006, Aromânii şi aromâna în conştiinţa contemporană [Les Aroumains et l'aroumain dans la conscience contemporaine], op. cit., p. 61.

22. Lors du recensement roumain de 2002, par exemple, plus de la moitié (14 258) des personnes qui se sont déclarées comme Aroumains (26387) ont déclaré l'aroumain comme langue maternelle.

23. En demandant le statut de minorité pour les Aroumains alors qu'ils ne sont pas autochtones en Roumanie, ils invoquent le cas des Albanais et Macédoniens de Roumanie, moins nombreux et pas plus autochtones, qui jouissent de ce statut dans la Constitution roumaine.

24. Thede KAHL, 2006, Istoria aromânilor, Bucureşti: Trictonic, pp. 229-318.

25. La séparation du roumain de l'aroumain aurait eu lieu au $\mathrm{VI}^{\mathrm{e}}$ siècle selon certains linguistes, au XIII ${ }^{e}$ siècle selon d'autres. Gustav Weigand fait remonter la rupture définitive du bloc commun au IX $\mathrm{x}^{\mathrm{e}}$ siècle, et c'est pour les $\mathrm{IX}^{\mathrm{e}}-\mathrm{X}^{\mathrm{e}}$ siècles que la plupart des linguistes se sont prononcés.

26. La plupart des auteurs de l'ouvrage collectif édité par l'Inalco en 1989 étaient des historiens (Les Aroumains, Cahiers du Centre d'étude des civilisations de l'Europe centrale et du Sud-Est, $\mathrm{n}^{\circ} 8$, présenté par Georges Castellan). Tant Neagu Djuvara que Petre Năsturel et Matei Cazacu, originaires de Roumanie, que l'Autrichien Max Demeter Peyfuss avaient esquissé des ouvertures qui n'ont pas eu de suite en Roumanie, où l'on est souvent revenu après 1990 à la position forgée à la fin du XIX siècle.

27. Rappelons par ailleurs que, opposés au statut de minorité pour les Aroumains en Roumanie, Matilda Caragiu Marioţeanu, Nicolae Saramandu ou encore Nicolae-şerban Tanaşoca ne vont jamais jusqu'à soutenir que les Aroumains de Bulgarie, Grèce, Albanie ou République de Macédoine seraient des Roumains. Ceux qui se réclament d'eux n'hésitent pas à franchir le pas; le mot qui revient le plus souvent pour préciser l'appartenance des Aroumains étant « Roumains du Sud».

28. IonuŢ NISTOR, 2009, „Problema aromână” în raporturile României cu statele balcanice (1903-1913), Iaşi: Editura UniversităŢii Alexandru Ioan Cuza, p. 273.

29. Enache TUşA, 2011, Imaginar politic şi identităŢi colective în Dobrogea, București: Institutul de ştiinţe politice şi relaţii internaţionale.

30. Alexandru GICA, 2009, "The Recent History of the Aromanians in Romania", in New Europe College Yearbook 2008-2009, Bucharest: New European College, pp. 173-200. URL : 
http://www.nec.ro/data/pdfs/publications/nec/2008-2009/ALEXANDRU_GICA.pdf (consulté le 13 mars 2014).

31. Irina NICOLAU, 1993, «Les caméléons des Balkans », Civilisations, vol. XIII, $\mathrm{n}^{\circ} 2$, p. $175-178$.

32. Voir aussi Corina IOSIF, 2013, «Frontières réelles, frontières imaginaires : l'identité aroumaine au début du Xxe siècle ", in Gilles ROUET et François soulages (dir.), Frontières géoculturelles \& géopolitiques, Paris : L’Harmattan, p. 133-147.

\section{RÉSUMÉS}

Les initiatives tous azimuts des Aroumains pour la promotion de leur langue et de leur culture après 1990 ont été plutôt bien accueillies au début en Roumanie. Le dépôt de la demande de reconnaissance de la minorité nationale aroumaine en 2005 va cependant provoquer une véritable levée des boucliers. Linguistes, historiens, hommes de lettres, réputés pour leurs travaux sur les Aroumains, souvent d'origine aroumaine, vont réagir vivement à ce tournant «national» de revendications identitaires qui couvaient depuis un moment. Comment interpréter cette réaction? Quel peut être son impact sur les études aroumaines dans ce pays?

The wide-ranging initiatives of the Aromanians to promote their language and culture after 1990 were received generally with sympathy at the beginning in Romania. On the other hand, the situation has changed radically with the application for recognition of the Aromanian national minority in 2005. The emergence of this national aspect of their cultural identity claims that smouldered long time ago, provoked strong public reactions of several linguists, historians and men of letters often of Aromanian extraction and renowned for their studies about Aromanian language and history. How to interpret these reactions? What impact they may have on Aromanian studies?

Numeroasele iniŢiative întreprinse de aromâni pentru promovarea limbii şi culturii lor după 1990 au fost mai degrabă bine primite în România. SituaŢia s-a schimbat însă radical odată cu înaintarea cererii de recunoaştere a minorităţii naţionale aromâne în 2005. Noua dimensiune naţională a unor revendicări identitare care mocneau de mult a provocat reacţii dure printre mulţi lingvişti, istorici şi scriitori adesea de origine aromână reputaŢi pentru contribuŢiile lor despre aromâni. Cum pot fi interpretate aceste reacţii? Ce impact pot avea ele asupra studiilor despre aromâni? 
INDEX

Index géographique : Roumanie

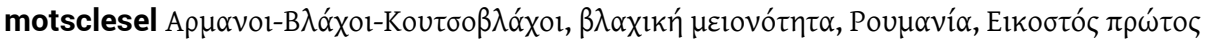

$\alpha \iota \omega \nu \alpha \varsigma, \Gamma \lambda \omega \sigma \sigma o \lambda o \gamma i ́ \alpha$

motsclestr Ulahlar, ulahlar azınlık, Romanya, Yirmi birinci yüzyılın

Keywords : Aromanians, aromanian minority, Romania, Twenty-first century, Linguistics

Mots-clés : Aroumains, Aroumains, minorité aroumaine, minorité aroumaine, Koutzo-Valaque,

Valaque

motsclesmk ВЛАСИ, ВЛАСИ МАЛЦИНСТВО, РОМАНИЈА, ДВАЕСЕТ И ПРВИОТ ВЕК, ЛИНГВИСТИКА

Thèmes : Linguistique

Index chronologique : vingt-et-unième siècle

\section{AUTEUR}

\section{NICOLAS TRIFON}

Écrivain, éditeur et sociolinguiste 